\title{
Effects of the application of the amniotic membrane in the healing process of skin wounds in rats ${ }^{1}$
}

Mariana Barbosa Dias Campelo', Joelita de Alencar Fonseca Santos", Antonio Luiz Martins Maia Filho'", Daniel Cabral Leão Ferreira'v, Luciana Barros Sant'Annav', Rauirys Alencar de Oliveiravı, Leonardo Fonseca MaiaVII, Emilia Ângela Loschiavo Arisawa ${ }^{\mathrm{VIII}}$

'Fellow PhD degree, Postgraduate Program in Biomedical Engineering, Universidade do Vale do Paraíba (UNIVAP), Sao Jose dos Campos-SP, Brazil. Conception, design, intellectual and scientific content of the study; acquisition and interpretation of data, technical procedures, manuscript preparation.

"Fellow PhD degree, Postgraduate Program in Biomedical Engineering, UNIVAP, Sao Jose dos Campos-SP. Assistant Professor, Nursing Department, Universidade Federal do Piauí (UFPI), Teresina-PI, Brazil. Technical procedures, manuscript preparation.

I'PhD, Associate Professor, Biotechnology and Biodiversity Laboratory, Universidade Estadual do Piauí (UESPI), Teresina$\mathrm{PI}$, Brazil. Technical procedures.

IV Veterinary, Biotechnology and Biodiversity Laboratory, UESPI, Teresina-PI, Brazil. Technical procedures.

${ }^{\vee} \mathrm{PhD}$, Immunology Laboratory, UNIVAP, Sao Jose dos Campos-SP, Brazil. Technical procedures.

VIPhD, Associate Professor, Department of Physiotherapy, UESPI, Teresina-PI, Brazil. Technical procedures.

VIIAssistant Professor, Department of Medicine, UFPI, Teresina-PI, Brazil. Technical procedures.

VIIIPhD, Biostimulation and Tissue Repair Laboratory, UNIVAP, Sao Jose dos Campos-SP, Brazil. Conception, design, intellectual and scientific content of the study; histopathological examinations; interpretation of data; critical revision.

\begin{abstract}
Purpose: To evaluate the efficacy of the application of the human amniotic membrane (HAM) on the inflammatory process, fibroblast proliferation, formation of collagenand reduction of skin wound areas in rats.

Methods: Thirty six rats were submitted to a surgical injury induction and divided into two groups ( $n=18)$ : group $C$ (control) and T (treated with the HAM). The macroscopic evolution in the wound area and the histological characteristics of the skin samples were evaluated.

Results: The regression of the wound area was greater in group $\mathrm{T}$. The histological analysis revealed a significant reduction $(p<0.05)$ in the inflammatory infiltrate in group $T$ at all experimental periods compared with that in the control group. Furthermore, the group $T$ presented a significant increase in the proliferation of fibroblasts at 14 and 21 days compared with group $C(p<0.05)$. Regarding the deposition of mature collagen fibers, there was an increase in the replacement of type III collagen by type I collagen in group $T(p<0.05)$.

Conclusion: Treatment with the HAM reduced the healing time as well as the inflammatory responses, increased the proliferation of fibroblasts, and induced a higher concentration of mature collagen fibers.
\end{abstract}

Key words: Wound Healing. Amnion. Collagen. Inflammation. Rats. 


\section{Introduction}

Chronic nonhealing skin wounds are one of the major health problems in several countries. It is estimated that 6 million individuals are affected by chronic wounds in the United States, with a cost estimated at more than 25 million dollars annually ${ }^{1}$

Growing advances have occurred in the treatment of skin lesions, considering the high availability of different coverages, whose development demands advanced technology and high costs. However, they are not completely effective in tissue regeneration and pain reduction ${ }^{2}$

Given the socioeconomic and clinical significance of skin wounds and their impact on public health, constant research is required to improve treatment protocols. Innovative solutions, particularly the application of extraembryonic stem cells derived from the placenta, chorion, and amniotic membrane, have been the subject of numerous studies in regenerative medicine and skin engineering, especially in recent years. The cells from these biomaterials are considered multipotent and have a high proliferation rate, good plasticity, and no immune response ${ }^{3}$

According to these studies, the human amniotic membrane (HAM), the inner layer of the fetal membranes, stands out for its ability to act in the healing process and reduce the tissue inflammatory response ${ }^{4}$. It should be emphasized that the use of the HAM has a long history in the treatment of wounds because since the first half of the last century, researchers have been studying its use in the reconstitution of tissue lesions, especially of the skin. In addition, it has been used in ophthalmology and in burns s-7 $^{5-7}$

As a part of the placenta, the HAM is discarded after birth; however, its application as a biological dressing or biomaterial has gained an importance owing to its low antigenicity, antimicrobial action, and ability to decrease exudates and adhesions, accelerate epithelization, reduce local pain, and act as a substrate for tissue growth ${ }^{5-8}$

The amniotic membrane, a tissue of an embryonic origin, is composed of three layers: a single epithelial layer, a thick basement membrane, and an avascular mesenchyme. Because it contains no nerves or lymphatic vessels, it is nourished and oxygenated by the chorionic fluid and amniotic fluid ${ }^{3}$.

The more internal epithelial layer consists of simple cuboidal epithelium that lines the amniotic membrane and is in direct contact with the amniotic fluid ${ }^{4}$. This layer is supported by the basal membrane, composed mainly of type IV collagen, elastin, fibronectin, laminin, and proteoglycans. The mesenchymal layer has three regions: an acellular layer that constitutes the main fibrous skeleton of the HAM, composed of types I and III collagen and fibronectin; a layer of mesenchymal cells similar to dispersed fibroblasts; and a randomly disposed sponge layer of collagen fibers separating the amniotic membrane from the chorion $^{7-9}$

Pre-clinical studies have employed the HAM successfully and showed that its use can accelerate the regeneration of various tissues ${ }^{5,10,11}$. However, only a limited number of studies have investigated its application as biological dressings.

It is important to increase our knowledge on the influence of the HAM on skin wounds in animal models, given that the efficacy and action of the HAM are not fully understood to promote the validation and improvement of therapeutic protocols. Thus, we hypothesized that the use of the HAM fragments as biological dressings can accelerate the tissue repair process. The objective of the present study was to evaluate the effectiveness of this biomaterial in the inflammatory process and fibrinogenesis, mainly of types I and III 
collagen, in different stages of the wound healing process in surgically induced skin lesions in rats.

\section{Methods}

This study was approved by the Research Ethics Committee Universidade do Vale do Paraíba (1.647.871) and the Committee of Ethics in Animal Experimentation of the Universidade Estadual do Piauí with $n^{\circ}$ 147.66-16.

Thirty-six 40-day-old male Wistar rats (Rattus norvegicus albinus) weighing $230 \pm$ $20 \mathrm{~g}$ were used and housed individually in polypropylene cages containing ration and water ad libitum in a 12/12-h light-dark cycle.

The animals were divided into two groups according to the experimental protocols: Control (C)- rats submitted to surgical injury induction and simulation of application of the HAM and Treated (T)- animals also submitted to surgical injury induction followed by the application of a fragment of the HAM. Thereafter, these groups were subdivided on the basis of the experimental periods of 7, 14, and 21 days, totaling six animals in each group.

\section{Harvesting and processing of the placenta}

Two human placentas were harvested and processed on the basis of the methodology described by Sant'Anna et al. ${ }^{11}$. Term human placentas were donated by patients with normal pregnancy (gestational age greater than or equal to 37 weeks and negative results for HIV-1 and 2, hepatitis B and C, and syphilis) who underwent elective cesarean sections and who signed the Informed Consent Form.

The placenta was placed in sterile plastic bags refrigerated at $10-15^{\circ} \mathrm{C}$, accommodated in thermal boxes with ice packs, and transported to the laboratory. The biomaterial was prepared in sterile conditions in a laminar flow hood, including the manual separation of the
HAM from the chorionic membrane, followed by washing with phosphate buffered saline (Sigma, St. Louis, MO, USA) containing 100$\mathrm{U} / \mathrm{mL}$ penicillin, $100-\mu \mathrm{g} / \mathrm{mL}$ streptomycin and amphotericin (Lonza, Basel, Switzerland) ${ }^{11}$. The HAM was then sectioned into pieces of an appropriate size $(4 \times 4 \mathrm{~cm})$, which was larger than the surgical wounds.

The fragments were stored separately, as reported by Hennerbichler et al. ${ }^{12}$, in 50$\mathrm{mL}$ vials filled with a solution containing glycerol and serum-free DMEM without phenol (volume 1:1) as a cryoprotectant agent and frozen at $-80^{\circ} \mathrm{C}$. The HAM was thawed immediately before use, until it reached an ambient temperature $\left(24^{\circ} \mathrm{C}\right)$, according to the procedure described by Baradaran-Rafii et al. ${ }^{13}$.

It is important to note that the HAM fragments were applied on the wound bed of the animals previously cleaned with $0.9 \%$ saline solution and with the stromal surface in contact with the bloody area, based on previous studies by Niknejad and Yasdanpanah ${ }^{9}$ who reported better results when this protocol was used.

\section{Experimental surgical procedures}

The animals were anesthetized with xylazine hydrochloride $(2 \%, 0.01 \mathrm{~mL} / \mathrm{kg})$ and ketamine hydrochloride $(10 \%, 0.005 \mathrm{~mL} / \mathrm{kg})$ intramuscularly after weighing. Next, a $6-\mathrm{cm}^{2}$ area on the dorsal region of the animal was shaved using an electric clipper, and local antisepsis was provided using $0.5 \%$ topical chlorhexidine alcohol. The surgical lesions, measuring $3 \mathrm{~cm}$ in diameter and $1 \mathrm{~cm}$ in depth, were created in a standardized manner with the help of a circular metal instrument with a metal blade ( $n \div 4)$ removing the entire thickness of the skin.

Immediately after surgical excision, the lesions of the animals in group $C(n=18)$ received no treatment, while those in group $T$ ( $n=18$ ) were covered with $4 \times 4 \mathrm{~cm}$ fragments 
of the HAM, exceeding its edges; the ends were secured in four points using methyl methacrylate.

After completing the surgical procedures, the rats in groups $\mathrm{C}$ and $\mathrm{T}$ were kept in individual cages and received the following treatments: antibiotic- amoxicillin $(0.001 \mathrm{~mL} / \mathrm{kg}$, intramuscularly, single dose); anti-inflammatory agent- flunixin meglumine (0.001 $\mathrm{mL} / \mathrm{kg}$, subcutaneously, 3 days); and analgesic- metamizole $(0.001 \mathrm{mg} / \mathrm{kg}$, orally, 3 days).

\section{Macroscopic analysis}

The wound area was macroscopically evaluated $0,7,14$, and 21 days after surgery using the images captured with a digital camera (Nikon Coolpix P100, with 10.3-megapixel resolution), without a flash and using natural light, fixed on a tripod at a distance of $20 \mathrm{~cm}$ from the wound. Macroscopic morphometry was performed using the software ImageJ to calculate the area of the wound at the different experimental periods; the results were expressed as the percentage of regression of the skin wound (area of the initial wound - area of the final wound / area of the initial wound $\times 100)^{14}$.

\section{Histological analysis}

At 7, 14, and 21 days after surgery, the animals in each group were euthanized by an overdose of anesthesia (Sodium Pentobarbital $100 \mathrm{mg} / \mathrm{kg}$, intraperitoneally) and disposed of in accordance with the guidelines established by the National Council for control of Animal Experimentation (CONSEA). The skin area where the wound was created and the surrounding area were removed and fixed in neutral buffered formalin (10\%, Synth, DiademSP, Brazil) and processed for routine histological processing. Thereafter, $5-\mu \mathrm{m}$ sections were stained with hematoxylin \& eosin (H\&E) and sirius red for the qualitative histological and morphometric analyses.

The H\&E-stained images_were scanned using the Leica DM 2500 microscope coupled to the Leica DFC 425 camera and Leica Application Suite Program LAS v3.7. Images were obtained from the cross sections of six sequential fields of each wound (40X objective) in a light microscope (final amplification $\times 400$ ). To quantify the number of inflammatory cells and fibroblasts, the images were analyzed using the software ImageJ, which enabled the elaboration of a grid and the individual marking of cell nuclei with the aid of the manual counting tool. The average score of 10 random microscopic fields per section was used to create a single score for each specimen in each experimental group.

The slides stained with picrosirius red were quantitatively evaluated via digital image analysis to calculate the area occupied by the deposition of types I and III collagen in the skin wound. The images were captured using the digital video camera (Leica DF425, Germany) coupled to the polarized light microscope (Leica DM2500) and digitalized in $1024 \times 768$ pixels and resolution of 24 bits/pixel with a general amplification of $x 100$. The images were processed using CellProfiler (Broad Institute of Harvard and MIT), which identified, isolated, and measured the areas occupied by type I and type III collagen in relation to the total area of the image. All histomorphometric analyses were performed blindly.

\section{Statistical analysis}

The statistical analysis was performed using the software Statistic 7.0. Statsoft ${ }^{\odot}$. The Kruskal-Wallis test was used for intra-group comparisons in the different experimental periods studied, while the Mann-Whitney test was used for the comparison of the groups. All levels of significance were set at $p<0.05$. 


\section{- Results}

\section{Macroscopic analysis}

The skin wounds of the animals were evaluated macroscopically $0,7,14$, and 21 days after the surgical procedures in terms of the formation of a scab and reduction in the wound area.

Among the animals in group $C$, no evidence of infection was observed 7 days after the surgical procedures, although the wounds were covered by extensive scabs (Figure 1-C7). At 14 days, the presence of granulation tissues was observed (Figure 1-C14), while at 21 days, the wound area showed a significant reduction, presenting a small and not yet re-epithelialized area (Figure 1-C21).

Conversely, infection was not also observed among the animals in group $T$ at 7 days owing to the application of the HAM, which remained adhered to the wound area, without forming a thick scab (Figure 1-T7). At 14 days, the animals had an advanced healing process, with a reduction in the surgical wound area and the presence of granulation tissues (Figure 1-T14). At 21 days, the wounds were practically closed and re-epithelialized, with no signs of the presence of the HAM fragment (Figure 1-T21).
Day 0
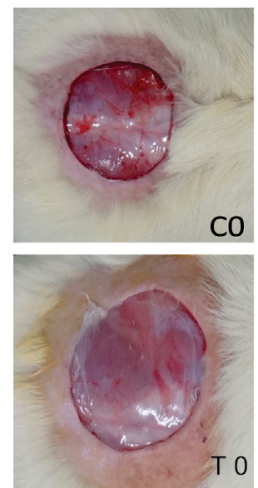

Day 7

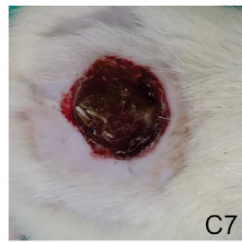

Day 14

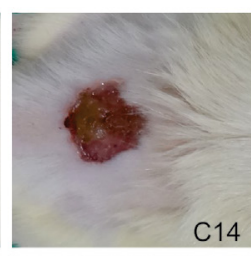

C14

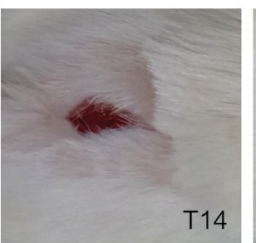

Day 21

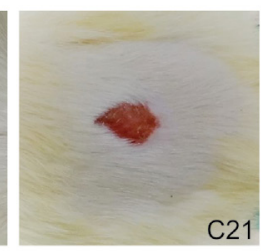

C21

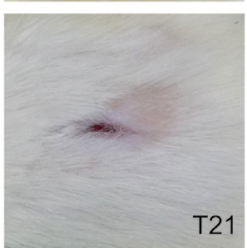

Figure 1 - Macroscopic images of the surgical wound area of the groups $C$ and T animals $0,7,14$, and 21 days after surgery.

\section{Microscopic analysis}

$\underline{7 \text { days }}$

The qualitative analysis of the H\&Estained slides on the $7^{\text {th }}$ day revealed the presence of superficial scabs in the group $C$ animals and an intense inflammatory response belowthescab (Figure2-C7) withapredominance of neutrophil polymorphonuclear cells and moderate vascular congestion in the hypodermis. The histological sections of group $T$ then revealed that the wounds treated with the HAM presented a discrete acute inflammatory infiltrate, with the beginning of the wound healing process characterized by an intense angiogenesis, increased deposition of extracellular matrix resulting from young and large fibroblasts (Figure 2-T7), and proliferation of type III collagen.

\section{4 days}

In the fragments of group $\mathrm{C}$, a mild inflammatory infiltrate was observed, with a predominance of lymphocytes, formation of granulation tissues, and discrete angiogenesis (Figure 2-C14). Conversely, in the histological specimens treated with the HAM, an improved organization of extracellular matrix was 
observed, with mild inflammatory response as well as the presence of polymorphonuclear leukocytes, absence of edema and vascular congestion, and presence of well-vascularized granulation tissues and organized collagen deposition (Figure 2-T14).

\section{1 days}

At 21 days, the specimens of group C showed a deposition of collagen fibers, with the formation of granulomas, which may have interfered with the wound healing process (Figure 2-C21). In the group treated with the HAM fragment, there was a closure of the wound area by second intention and an organization of the collagen fibers (Figure 2-T21). In this group, proliferation of keratinized squamous epithelium, presence of fibroblasts, and discrete vascularization, were observed.

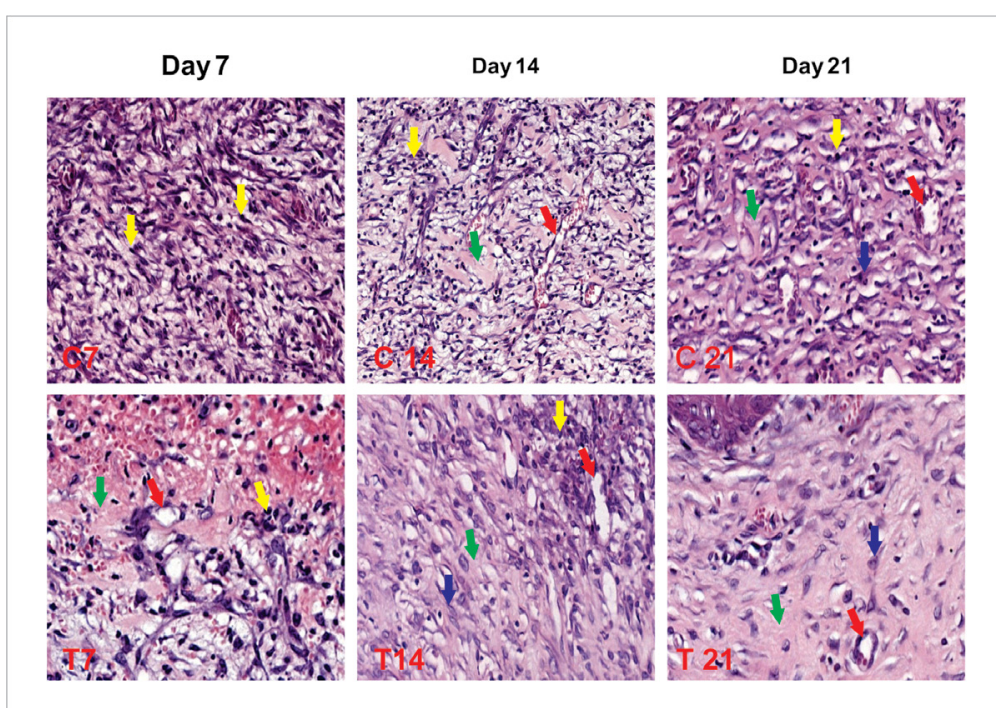

Figure 2 - Qualitative histological aspects of the wound healing process. Note the inflammatory response and proliferation of fibroblasts and collagen fibers in the region of the wound in groups $\mathrm{C}$ (control) and $\mathrm{T}$ (treated with the human amniotic membrane) at 7, 14, and 21 days (Hematoxylin \& Eosin, x400). Yellow arrows indicate the inflammatory cells; red arrows, the blood vessels; blue arrows, the fibroblasts; and green arrows, the collagen fibers.

\section{Analysis of the collagen fibers}

During the wound healing process, type III collagen (immature) is replaced by type I collagen. The qualitative analysis of the sirius red-stained slides showed the progression of this process in both groups throughout the experiment.

The analysis of the images captured using the polarized light microscope in the control group showed a predominance of type III collagen (immature) as shown in Figures 3A (non-polarized) and 3B (polarized) at 14 days and in Figures $3 C$ (non-polarized) and 3D (polarized) at 21 days.
Conversely, in the samples of the HAM-treated group (T) at 14 days, there was a concentration of the subtypes of partially organized types I and III collagen, with an evident prevalence of type III collagen, both in the microscopic images of the non-polarized light (Figure 3E) and polarized light (Figure 3F). At 21 days, the histological sections showed that the wound area was fully re-epithelialized and that types I and III collagen were aligned and fully organized, with a predominance of type I collagen as shown in Figures $3 G$ (nonpolarized) and $3 \mathrm{H}$ (polarized), and an advanced collagen fiber remodeling. 


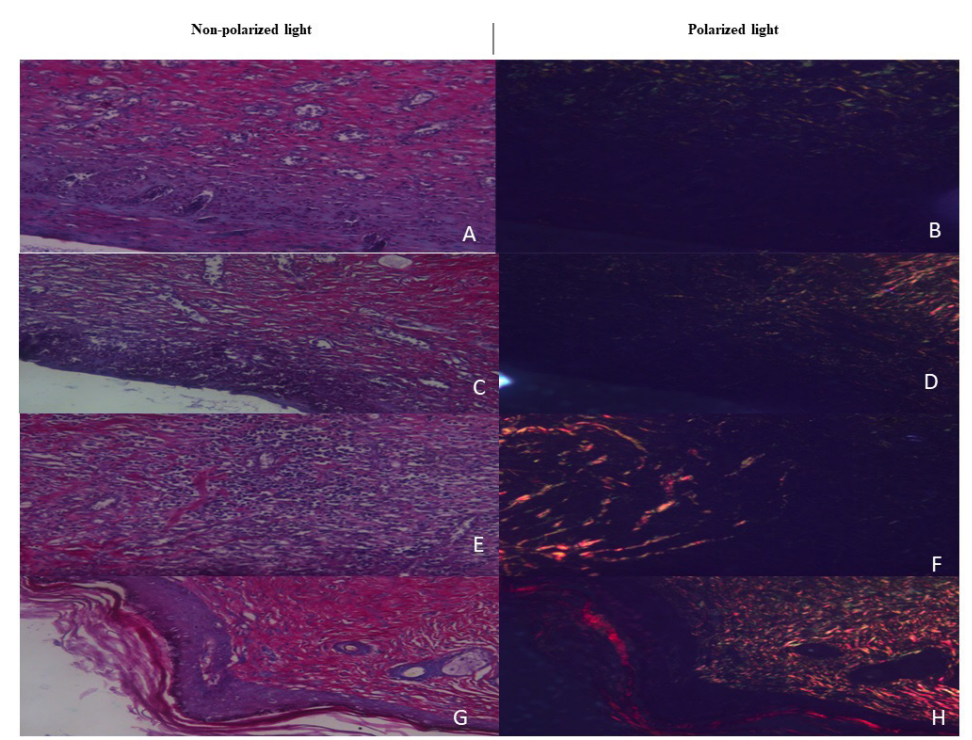

Figure 3 - Qualitative aspects of the collagen fibers in groups $C$ (control) and T (treated with the human amniotic membrane) at 14 and 21 days, stained with sirius red (x100)

Macroscopic analysis of the percentage of regression of the areas of cutaneous lesions

The macroscopic analysis of the percentage of regression of the wounds showed a significant increase in group $T$ when compared with that in group $C$. On the $7^{\text {th }}$ day and $21^{\text {st }}$ day, a statistically significant difference was observed ( $p<0.05)$ with respect to the areas of regression of the wound in group T compared with that in the control group. However, at 14 days, there was no significant difference between groups $\mathrm{C}$ and $\mathrm{T}$ (Figure 4).

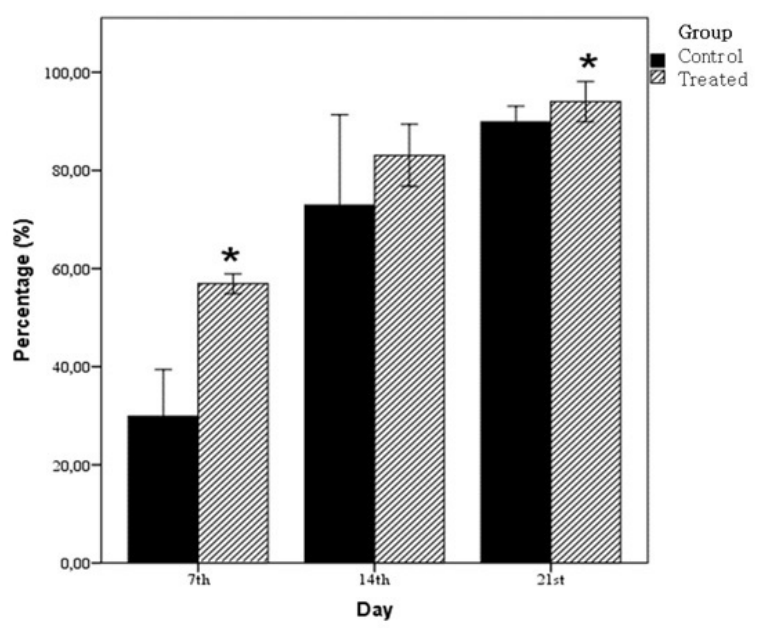

Figure 4 - Percentage of regression of the surgical wounds in groups $\mathrm{C}$ (control) and $\mathrm{T}$ (treated with the human amniotic membrane) at 7, 14, and 21 days. The result of the percentage of regression of the skin wound was defined using the following formula: ([area of the initial wound - area of the final wound] / [area of the initial wound] $\times 100)$; Mann-Whitney $U$ test. The values of a statistical significance are indicated by $*(p<0.05)$. 
Quantitative analysis of the inflammatory cells and fibroblasts

There was a reduction in the inflammatory infiltrate in group $\mathrm{T}$ compared with that in group $C$. The intergroup analysis showed a statistically significant difference $(p<$
0.05 ) at 7,14 , and 21 days. Group T showed a significant increase in the number of fibroblasts $(p<0.05)$ when compared with group C only on the $14^{\text {th }}$ and $21^{\text {st }}$ days of the wound healing process. However, no significant difference was observed in the number of fibroblasts between both groups at 7 days (Figure 5).

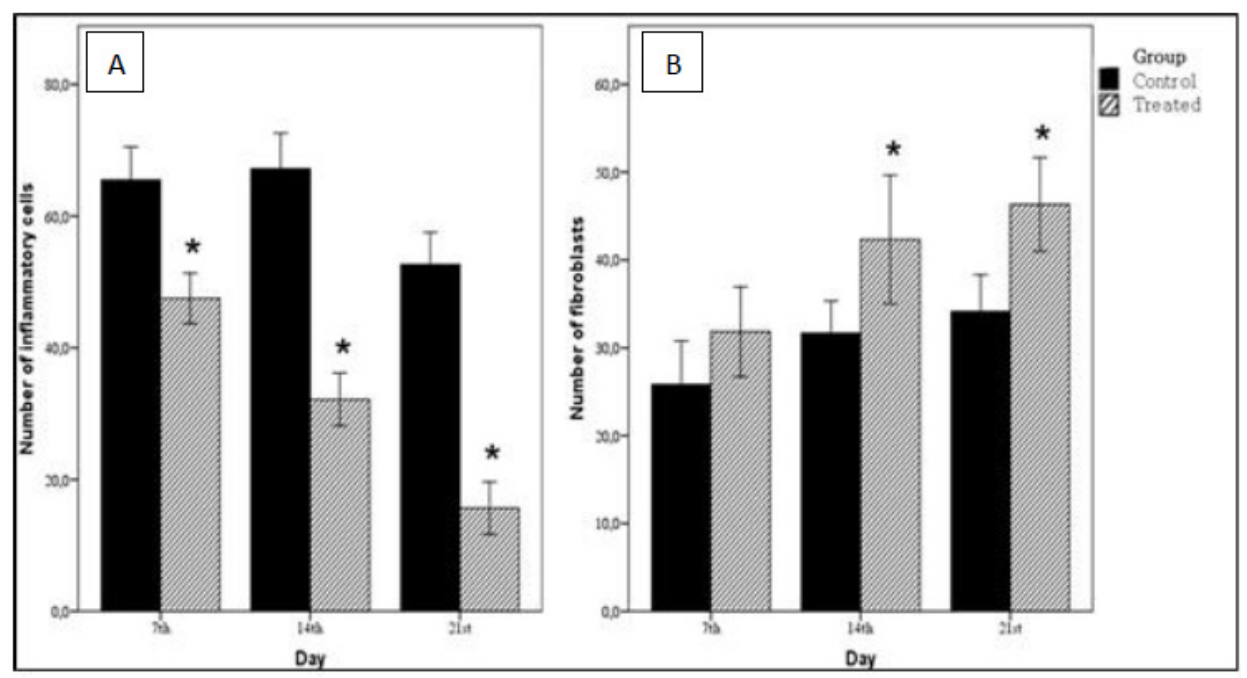

Figure 5 - Histological analysis of groups $C$ (control) and T (treated with the human amniotic membrane) at 7, 14 , and 21 days. (A) Number of inflammatory cells $\left(20.000 \mu \mathrm{m}^{2}\right)$ and (B) number of fibroblasts $\left(20.000 \mu \mathrm{m}^{2}\right)$; Mann-Whitney $U$ test. The values with a statistical significance are indicated by $*(p<0.05)$.

\section{Quantitative analysis of the collagen concentration}

Regarding the percentage of types I and III collagen present in the wound healing process at 7 days, the average percentage of types I and III collagen was practically equal in both groups, with a predominance of the latter. However, at 14 days, there was a statistically significant increase in the replacement of type III collagen by type I collagen in group $T$ compared with that in group $C(p \leq 0.05)$. At 21 days, a similar distribution of types I and III collagen subtypes was observed in both groups, with no statistical significance (Figure 6).

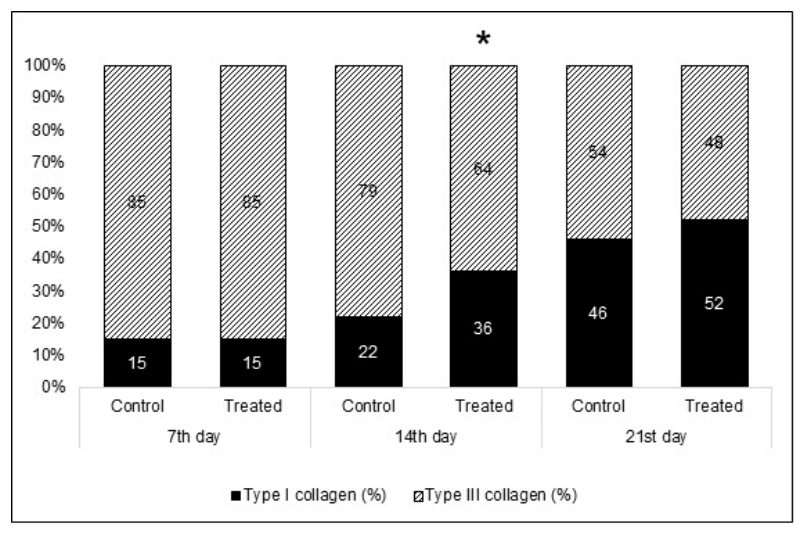

Figure 6 - Percentage of types I and III collagen in the surgical wounds of groups $C$ (control) and $T$ (treated with HAM) at 7, 14, and 21 days; MannWhitney $U$ test, *statistically significant at $p<0.05$. 
In the present study, the typification of the collagen fibers via the analysis of the polarized light allowed us to observe a balance between the distribution of types I (yellow, orange, or red) and III collagen (green) from the margins to the center of the wound.

\section{- Discussion}

The amniotic membrane acts as a transplanted basal membrane that serves as a substrate for epithelization ${ }^{3}$. Recent studies have revealed that the HAM is a rich source of MSCs, collagen matrix, and growth factors. In addition, it serves as a support for tissue repair and regeneration ${ }^{15}$.

The objective of this study was to evaluate the efficacy of the application of the HAM regarding the inflammatory response, fibrinogenesis, and remodeling and organization of collagen during the wound healing process; thus, the periods of 7,14 , and 21 days were selected, allowing us to evaluate this process ${ }^{14}$

The results showed that the application of the HAM accelerated the wound healing process, with closure of the injury. The antiinflammatory action of the HAM reduced the duration of the initial healing phase, favoring the early initiation of the formation of collagen fibers, as in the remodeling phase, characterized by the replacement of type III collagen (immature) by type I collagen (mature).

After tissue injury, the inflammatory response plays an important role in the processes of normal and pathological healing because it provides protection against invading pathogens and removes damaged tissues ${ }^{16}$. However, a prolonged inflammatory response determines a delay in the progression in the different phases of the wound healing process $^{14-17}$ The results obtained in this study showed that the HAM played a protective role in the wound area, providing a favorable microenvironment to repair the skin, compared with the results of the control group. The histological analysis demonstrated that the inflammatory response in group $T$ was less intense at all stages of the process than that in group $\mathrm{C}$, favoring a more organized progression. The considerable decrease in the number of inflammatory cells at 14 days and 21 days in group T demonstrated that the HAM did not increase the inflammatory response in the wounds, which corroborates their low immunogenicity and its possible allogeneic use $^{18-19}$

Manuelpillai et al. ${ }^{14}$ reported that the anti-inflammatory properties are associated with the presence of several factors in the stroma of the HAM, such as interleukin (IL)10, IL-1 receptor antagonist (IL-1), hyaluronic acid, and prostaglandin E2 (10). Tseng et al. ${ }^{20}$ reported that the anti-inflammatory activity of the HAM requires a close contact with its stromal matrix. The high content of proteoglycans and glycoproteins of the intermediate layer of the stromal matrix contains a non-fibrillar mesh, mainly of type III collagen ${ }^{6}$. In addition, the microenvironment maintained with the use of fragments allows the receptor tissue of this biomaterial to use all growth factors and other products present to potentiate the development of tissue repair.

The results obtained showed that during the proliferative phase ( 7 to 14 days), the number of fibroblasts increased in both groups studied. However, the histomorphometric analysis showed a considerable increase in the number of these cells in group $\mathrm{T}$, which was probably related to the presence of several growth factors in the epithelium of the membrane. The HAM has the ability to stimulate skin repair and synthesize essential growth factors and cytokines that promote cell migration, proliferation, and metabolic activity in the cells and tissues ${ }^{21-22}$. 
It is evident that the repair process had an early initiation in the wounds protected compared with that in the fully exposed wounds because the membrane acts as a biological bandage protecting the injured tissues; this favors local hydration and avoids contamination by microorganisms and contaminated materials ${ }^{5}$.

Meller et al. ${ }^{6}$, Tahan and Tahan $^{8}$, and Sant'Anna et $a .^{23}$ demonstrated that the HAM exerts beneficial actions in the repair and regeneration of tissues owing to its antiinflammatory, analgesic, anti-bacterial, reepithelization, and healing effects on wounds.

Histological staining by picrosirius red is the method of choice to evaluate the presence of collagen fibers, staining them red. These fibers, when visualized under an optical microscope with polarizing lens, assume different colors. They become orange if they are type I collagen or greenish if they are type III collagen. This birefringence is highly specific for collagen ${ }^{24}$.

Histologically, the dermis of rodents is composed of dense connective tissue formed by types I and III collagen and a small amount of elastic fibers. The proliferative phase of healing occurs 3 to 5 days after tissue injury and is characterized by an increase in the number of fibroblasts, which produce and deposit a large amount of type III collagen. Later, the maturation of these fibers occurs, with their remodeling and replacement by type I collagen, which is thicker and more resistant ${ }^{25}$. The present study demonstrates that the collagen fibers of the group $T$ animals reached maturity and early macromolecular orientation compared with that of the control animals, which had a slower wound healing process.

Duarte et $a l .{ }^{5}$ reported that in the wound healing process of rats treated with the HAM, a predominance of mature collagen fibers was observed. Vascular formation was more significant in the animals that received the HAM, indicating that even its conservation at lower temperatures does not inactivate proangiogenic substances previously identified in the cryopreserved membrane ${ }^{26}$.

In addition, the harvesting of the amniotic membrane does not offer any risk to the newborn or the parturient, and the membrane can be easily obtained and stored in laboratories for later use. The use of these biomaterials does not raise ethical or religious conflicts, besides being a cost-effective alternative, when compared to the isolation and culture of stem cells, which require more complex processes ${ }^{4-18}$.

Therefore, the results confirm the biocompatibility of the HAM and that its application promoted a greater formation of collagen fibers and total re-epithelialization of the wound area. We emphasize that the use of the HAM offers the advantages of high availability, ease of application, and good storability $^{12-18}$. In this context, it is possible to affirm that by presenting properties that favor the re-structuring of the damaged portions of the tissues, the amniotic membrane could be used as a biological coverage of acute and chronic wounds ${ }^{18-26}$.

\section{Conclusions}

The application of human amniotic membrane fragments modulated the cascade of biological events from extensive tissue injuries, reduced wound regression and inflammatory response, and increased fibroblast proliferation and early stimulation of the production and organization of collagen fibers. Besides meeting the functional goals of the reconstructed skin, the results obtained are important given the reduction in the total time of the wound healing process and the final appearance observed. 


\section{References}

1. Tamama K, Kerpedjieva SS. Acceleration of wound healing by multiple growth factors and cytokines secreted from multipotential stromal cells/mesenchymal stem cells. Adv Wound Care. 2012;1(4):177-82. doi: 10.1089/wound.2011.0296.

2. Lorenzo Hernández MP, Hernández Cano RM, Soria Suárez MI. Heridas crónicas atendidas en un servicio de urgencias. Enfermería Glob. 2014;13(3):23-31.

3. Toda A, Okabe M, Yoshida T, Nikaido T. The potential of amniotic membrane/amnionderived cells for regeneration of various tissues. J Pharmacol Sci. 2007;105(3):215-8. doi: 10.1254/jphs.CR0070034.

4. Niknejad $H$, Peirovi $H$, Jorjani $M$, Ahmadiani A, Ghanavi J, Seifalian AM. Properties of the amniotic membrane for potential use in tissue engineering. Eur Cell Mater. 2008;15(April):88-9. doi: 10.22203/eCM. v015a07.

5. Duarte IGL, Duval-Araujo I. Amniotic membrane as a biological dressing in infected wound healing in rabbits. Acta Cir Bras. 2014;29(5):334-9. doi: 10.1590/ S0102-86502014000500008.

6. Meller $D$, Pauklin $M$, Thomasen $H$, Westekemper $\mathrm{H}$, Steuhl K-P. Amniotic membrane transplantation in the human eye. Dtsch Arztebl Int. 2011;108(14):243-8. doi: 10.3238/arztebl.2011.0243.

7. Mamede A, Carvalho M, Abrantes A, Laranjo M, Maia C, Botelho M. Amniotic membrane: from structure and functions to clinical applications. Cell Tissue Res. 2012;349(2):447-58. doi: 10.1007/s00441012-1424-6.

8. Tahan AC, Tahan V. Placental amniotic epithelial cells and their therapeutic potential in liver diseases. Front Med (Lausanne). 2014;1:48. doi: 10.3389/ fmed.2014.00048.

9. Niknejad H, Yazdanpanah G. Opposing effect of amniotic membrane on angiogenesis originating from amniotic epithelial cells. J Med Hypotheses Ideas. 2014;8(1):39-41. doi: 10.1016/j.jmhi.2013.08.002.

10.Manuelpillai U, Moodley Y, Borlongan C V., Parolini O. Amniotic membrane and amniotic cells: potential therapeutic tools to combat tissue inflammation and fibrosis?
Placenta. 2011;32(Suppl. 4):S320-5. doi: 10.1016/j.placenta.2011.04.010

11.Sant'Anna LB, Cargnoni A, Ressel L, Vanosi G, Parolini O. Amniotic membrane application reduces liver fibrosis in a bile duct ligation rat model. Cell Transplant. 2011;20(3):44153. doi: $10.3727 / 096368910 \times 522252$.

12. Hennerbichler S, Reichl B, Pleiner D, Gabriel C, Eibl J, Redl H. The influence of various storage conditions on cell viability in amniotic membrane. Cell Tissue Bank. 2007;8(1):1-8. doi: 10.1007/s10561-0069002-3.

13.Baradaran-Rafii A, Aghayan H-R, Arjmand $B$, Javadi M-A. Amniotic membrane transplantation. J Ophthalmic Vis Res. 2007;2(1):58-75.

14.Estevão LRM, Mendonça FDS, BaratellaEvêncio L, Simões RS, Barros MEG De, Arantes RME, Rachid MA, Evêncio-Neto J . Effects of aroeira (Schinus terebinthifoliu Raddi) oil on cutaneous wound healing in rats. Acta Cir Bras. 2013;28(3):202-9. doi: 10.1590/S0102-86502013000300008.

15.Gibbons GW. Grafix , a cryopreserved placental membrane, for the treatment of chronic/stalled wounds. Adv Wound Care. 2015;4(9):534-44. doi: 10.1089/ wound.2015.0647.

16.Qian LW, Fourcaudot AB, Yamane K, You T, Chan RK, Leung KP. Exacerbated and prolonged inflammation impairs wound healing and increases scarring. Wound Repair Regen. 2016;24(1):26-34. doi: 10.1111/wrr.12381.

17.Demidova-Rice TN, Hamblin MR, Herman IM. Acute and impaired wound healing: pathophysiology and current methods for drug delivery, part 1: normal and chronic wounds: biology, causes, and approaches to care. Adv Skin Wound Care. 2012;25(7):30414. doi: 10.1111/wrr.12381.

18. Nicodemo $M$ de $C$, Neves LR das, Aguiar JC, Brito F de S, Ferreira I, Sant'Anna LB, Raniero LJ, Martins RAL,Barja PR, Arisawa EALS. Amniotic membrane as an option for treatment of acute Achilles tendon injury in rats. Acta Cir Bras. 2017 Feb;32(2):125-39. doi: 10.1590/s0102-865020170205.

19.Duan-Arnold Y, Uveges TE, Gyurdieva A, Johnson A, Danilkovitch A. Angiogenic potential of cryopreserved amniotic membrane is enhanced through retention 
of all tissue components in their native state. Adv Wound Care. 2015;4(9):513-22. doi: 10.1089/wound.2015.0638.

20.Tseng SCG, Espana EM, Kawakita T, Di Pascuale MA, Li W, He H, Liu TS, Cho TH, Gao YY, Yeh LK, Liu CY. How does amniotic membrane work? Ocular Surface. 2004;2:177-87.doi: 0124(12)70059-9.

21.Frykberg RG, Banks J. Challenges in the treatment of chronic wounds. Adv Wound Care. 2015;4(9):560-82. doi: 10.1089/ wound.2015.0635.

22.Gutiérrez-Moreno $S$, Alsina-Gibert $M$, Sampietro-Colom L, Pedregosa-Fauste S, Ayala-Blanco P. Estudio coste-beneficio del trasplante de membrana amniótica para úlceras venosas de extremidades inferiores refractarias a tratamiento convencional. Actas Dermosifiliogr. 2011;102(4):284-8. doi: 10.1016/j.ad.2011.01.003.

23.Sant'Anna LB, Brito FS, Barja PR, Nicodemo MC. Long-term effects of human amniotic membrane in a rat model of biliary fibrosis.
Brazilian J Med Biol Res. 2017;50(7):e5692. doi: 10.1590/1414-431X20175692.

24.Junqueira LCU, Bignolas G, Brentani RR. Picrosirius staining plus polarization microscopy, a specific method for collagen detection in tissue sections. Histochem J. 1979;11(4):447-55. doi: 10.1007/ BF01002772.

25.Baptistella E, Malafaia O, Czeczko NG, Ribas-Filho JM, Nassif PAN, Nascimento MM do, Pachnicki, JPA. Comparative study in swines' vocal cords healing after excision of fragment with $\mathrm{CO}_{2}$ laser with mitomycin and 5-fluorouracil postoperative topical application. Acta Cir Bras. 2009 JanFeb;24(1):13-8. PMID: 19169536.

26. Barbuto RC, de Araujo ID, Bonomi DO, Tafuri LSA, Neto AC, Malinowski R, Bardin VSS, Leite MD, Duarte IGL. Use of the amniotic membrane to cover the peritoneal cavity in the reconstruction of the abdominal wall with polypropylene mesh in rats. Rev Col Bras Cir. 2015;42(1):49-54. doi: 10.1590/0100-69912015001010.

\section{Correspondence:}

Mariana Barbosa Dias Campelo

Departamento de Enfermagem, UFPI

Campus Universitário Ministro Petrônio Portela

Avenida Nossa Senhora de Fátima, Bloco SG 12

64.049-550 Teresina - PI Brasil

Tel.: (55 86)3215-5881

marianadias@hotmail.com

Received: Oct 10, 2017

Review: Dec 12, 2017

Accepted: Jan 11, 2018
Conflict of interest: none

Financial source: none

${ }^{1}$ Research performed at Laboratory of Experimental Surgery, and Laboratory of Core Research in Biotechnology and Biodiversity (NPBIO), Faculty of Medical Sciences, Universidade Estadual do Piauí (UEPI), Teresina-PI, Brazil. Part of PhD degree thesis, Postgraduate Program in Biomedical Engineering, Universidade do Vale do Paraíba (UNIVAP). Tutor: Profa. Emilia Ângela Loschiavo Arisawa. 